\title{
Electroretinographic studies following vitrectomy and intraocular silicone oil injection*
}

\author{
TRAVIS A MEREDITH, ${ }^{1}$ DELWIN T LINDSEY,${ }^{2}$ HENRY F EDELHAUSER, ${ }^{2}$ \\ AND ARNOLD I GOLDMAN ${ }^{2}$
}

From the 'Department of Ophthalmology, Emory University, 1365 Clifton Road, NE, Atlanta, Georgia 30322, and the ${ }^{2}$ Department of Ophthalmology, Medical College of Wisconsin, $8700 \mathrm{~W}$ Wisconsin Avenue, Milwaukee, Wisconsin 53226, USA

SUMMARY Vitrectomy was performed bilaterally in pigmented rabbits and followed by silicone oil injection in one eye only. Electroretinographic studies were performed preoperatively and at selected postoperative intervals. In the early postoperative period both eyes showed an initial reduction in $\mathrm{a}$ and $\mathrm{b}$ wave amplitudes, followed by a return to baseline values. The amplitudes were symmetrically and equally affected. Sequential studies over a 20 -month period showed no deterioration in electroretinographic response in eyes with and without silicone oil injection.

Silicone oil injection into the vitreous cavity in the management of complicated retinal detachment was introduced in the United States by Cibis in $1961 .{ }^{12}$ Numerous early laboratory studies indicated that the eye tolerated silicone oil well. ${ }^{3-6}$ Despite initial clinical enthusiasm a high rate of failure and complication was subsequently reported in human eyes. ${ }^{7}$ Cataract, glaucoma, and keratopathy can be major anterior segment problems after silicone oil injection. ${ }^{78}$ Of more importance potentially was the suggestion in a series of animal experiments that silicone oil causes a form of retinopathy marked by early and lasting reduction in electroretinographic (ERG) amplitude, accompanied by retinal degeneration. ${ }^{9-11}$ A decline in ERG amplitude may be due, however, to the insulating properties of the silicone oil.

While falling into disuse in the United States, the technique of silicone oil injection was further refined and advanced by Scott in England. ${ }^{12-15}$ Encouraging results were also reported by Okun, ${ }^{16}{ }^{17}$ Grey and Leaver, ${ }^{18}$ and Zivonjnovic and associates. ${ }^{19}$ Anterior segment complications appeared to be important in subsequent visual loss in studies by Leaver and Grey, ${ }^{8}$ but these difficulties are conceivably preventable by removal of the silicone oil. The question of

*Presented in part before the Association for Research in Vision and Ophthalmology, Sarasota, Florida, 1 May 1981.

Correspondence to Travis A Meredith, MD, Department of Ophthalmology, Emory University, 1365 Clifton Road, NE, Atlanta, Georgia 30322 USA. the existence of a silicone oil retinopathy remains critical. Recent studies have demonstrated no significant decline in postoperative electroretinographic responses in the first several weeks after silicone oil injection into the vitrectomised rabbit eye..$^{2021}$ This study extends these observations over a significantly longer period.

\section{Materials and methods}

Thirteen New Zealand pigmented rabbits weighing 3.0 to $4.0 \mathrm{~kg}$ were studied. One to two weeks before the start of the experiments five to eight cryotherapy applications were placed in the nasal retina to produce an area of chorioretinal adhesion through which an incision could be made for insertion of the vitrectomy instrument. ${ }^{22}$ General anaesthesia consisted of intramuscular injections of ketamine 1.0-2.0 $\mathrm{ml}$ and acepromazine $1-2 \mathrm{mg} / \mathrm{kg}$ for all operative procedures.

Preoperatively and one to two weeks later electroretinograms were recorded. Photic stimulation was supplied by a Grass PS-22 photostimulator with the flash unit placed perpendicular to the visual axis and 6 inches $(15 \mathrm{~cm})$ from each eye. Responses were sensed by a bipolar corneal electrode of conventional design (Burian Allen), differentially amplified (Tektronix 7A22; $3 \mathrm{db}$ points (points beyond which $50 \%$ of the signal was attenuated to reduce noise within the system): $0.1300 \mathrm{~Hz}$ ), and displayed on the face of a storage oscilloscope (Tektronix 7313). 
The rabbits were kept in the darkened test room for at least 30 minutes prior to evaluation. Recordings were made with the rabbits held in a special plastic restraining box. The eyes were topically anaesthetised with a $0.5 \%$ solution of proparacaine hydrochloride prior to insertion of the corneal electrode; otherwise the rabbits remained unmedicated throughout the recording session.

Each eye was evaluated at two flash radiances: maximum photostimulator radiance (I 16) and approximately one-quarter maximum (I 4). All electroretinographic data presented are based on the averages of two responses at each flash radiance.

Under repeat anaesthesia a vitrectomy was performed bilaterally on the same day in 12 rabbits. In the 13th animal surgery was performed in one eye only. In addition to intramuscular ketamine and acepromazine $1 \mathrm{ml}$ of lignocaine $1 \%$ was injected retrobulbarly for each eye. Pupils were dilated prior to surgery with cyclopentolate $1 \%$ and phenylephrine hydrochloride $10 \%$, two drops each to each eye.

Initial incisions were carried through previously treated inferonasal retina with a sclerotome. The Machemer vitreous infusion suction cutter (VISC) with light source was placed through the incision and a pre-placed 5-0 Mersilene suture tied around it to render the incision water tight. Ringer's lactate infusion was used in all eyes. An attempt was made to remove as much vitreous gel as possible; to this end a tiny drop of fluorescein was injected into many eyes to provide greater demarcation of the gel. All surgery was performed under microscopic control.

Silicone oil was injected into only one eye of the two operated eyes using a 16 gauge cannula; the fellow operated eye served as a control. Medical grade silicone oil of 1000 centistokes viscosity, supplied through the courtesy of Mr Peter Leaver and Dr Robert Machemer, was used in all cases. The amount injected varied from 1.0 to $1.9 \mathrm{ml}$, producing an estimated fill of the vitreous cavity of $60-90 \%$. In most cases approximately $80-90 \%$ of the vitreous cavity appeared to be filled with silicone oil. Post- operative clinical examinations and sequential electroretinograms were recorded from day one postoperatively and continued at selected intervals for 20 months.

One eye with silicone oil injection developed a retinal detachment after one month; the fellow eye was followed up for two months. One eye undergoing vitrectomy alone developed a retinal detachment three months after surgery; the fellow eye was followed up for nine months.

Histological examinations of both eyes were performed at the following time intervals: four days (1); two months (1); three months (1); six months (2); nine months (1). To obtain the specimens the animals were deeply anaesthetised, and their eyes were enucleated; they were then immediately killed. Immediately after enucleation the eyes were placed in a fixative consisting of $1 \%$ glutaraldehyde, $1 \%$ formaldehyde in $0.1 \mathrm{M}$ phosphate buffer, $\mathrm{pH} 7 \cdot 0$ $7 \cdot 2$, at room temperature $\left(20^{\circ} \mathrm{C}\right)$. After one minute an incision approximately $1 \mathrm{~cm}$ in length was made along the pars plana to facilitate penetration of fixative. One hour later the anterior segment was removed, and the tissue was allowed to fix overnight at $4^{\circ} \mathrm{C}$. Finally the tissue was washed in buffer, dehydrated, and embedded in epoxy resin. Semithin sections $(1 \mu \mathrm{m})$ were cut on a DuPont Sorvall Porter Blum MT-2B ultramicrotome, mounted on glass slides, and stained with toluidine blue for light microscopy.

\section{Results}

The average preoperative $b$ wave value was 161 microvolts for eyes undergoing vitrectomy alone and 170 microvolts for eyes subjected to vitrectomy and silicone oil injection (Table 1). Two days postoperatively there was equal and symmetrical decrease in $b$ wave amplitudes in both sets of eyes (Table 1 and Fig. 1). Eyes undergoing vitrectomy alone decreased $31 \%$ $(\Delta \mathrm{B}=-50$ microvolts); eyes with vitrectomy and silicone oil injection declined $39 \%(\Delta B=-67$ micro-

Table 1 b wave amplitude (microvolts)

\begin{tabular}{clllll}
\hline & \multicolumn{2}{l}{ Vitrectomy } & & \multicolumn{2}{l}{ Vitrectomy + silicone oil } \\
\cline { 2 - 3 } \cline { 5 - 5 } \cline { 5 - 5 } & No. of animals & Avg. value $\pm S E$ & & No. of animals & Avg. value $\pm S E$ \\
\hline Preoperative & 12 & $161 \pm 9 \cdot 6$ & 13 & $169 \pm 13 \cdot 2$ \\
Postoperative & 5 & $116 \pm 17 \cdot 8$ & 6 & $113 \pm 16$ \\
Day 2 & 5 & $166 \pm 31 \cdot 7$ & 6 & $122 \pm 21$ \\
5 & 7 & $191 \pm 16 \cdot 3$ & 7 & $233 \pm 27$ \\
14 & 4 & $258 \pm 34$ & 3 & $188 \pm 21 \cdot 2$ \\
30 & 7 & $188 \pm 16 \cdot 7$ & 8 & $201 \pm 14$ \\
Month 2 & 6 & $213 \pm 26$ & 7 & $160 \pm 14$ \\
3 & 4 & $176 \pm 39$ & 6 & \\
\hline
\end{tabular}




\section{Vitrectomy vs Vitrectomy + Silicone Oil}

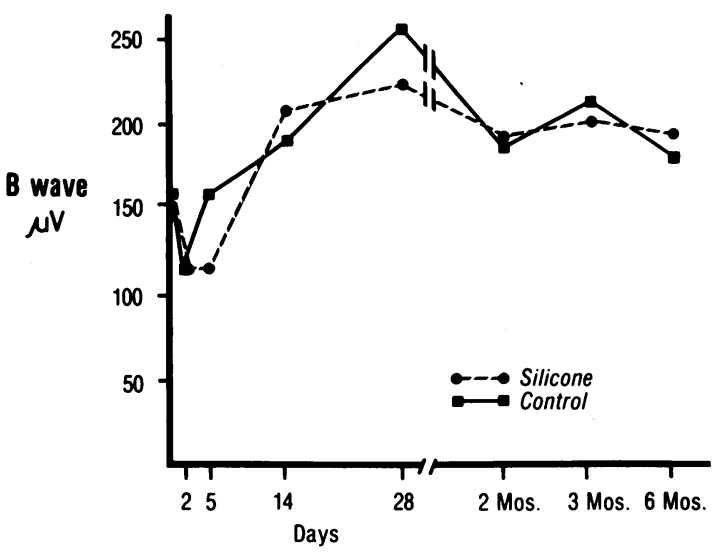

Fig. 1 b Wave amplitudes are plotted against postoperative time.

volts) (Fig. 2). By the seventh postoperative day eyes with vitrectomy alone showed a rise in $b$ wave amplitude of $25 \% \quad(\Delta B=41$ microvolts $)$ over baseline values (Figs. 3-8). Eyes undergoing vitrectomy and silicone oil injection rose $35 \%(\Delta B=60$ microvolts) compared with baseline preoperative values. Values in both groups of eyes tended to remain slightly above baseline for a six-month period of observation, showing a slow shift back to near baseline. In some

\section{Vitrectomy vs Vitrectomy + Silicone Oil}

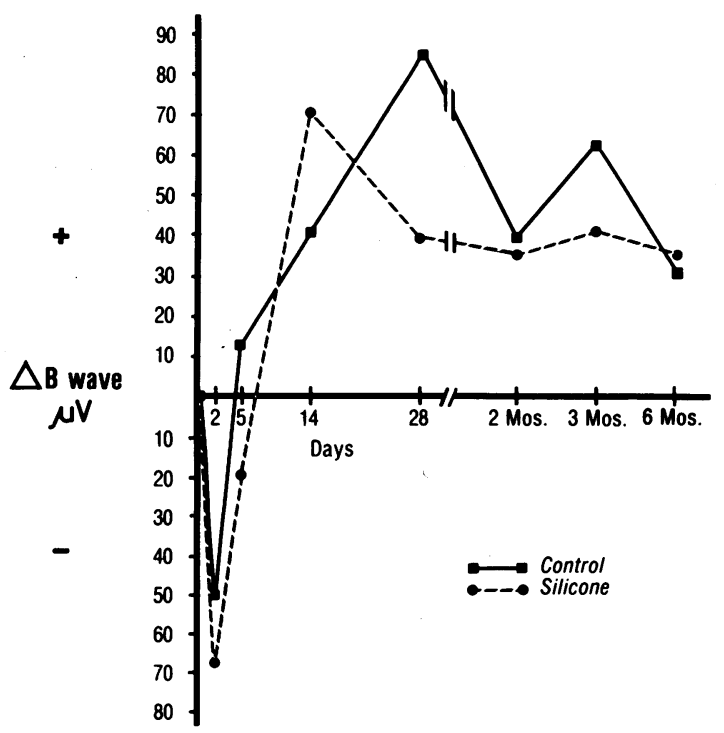

Fig. 2 Changes in b wave amplitudes are plotted against postoperative time.

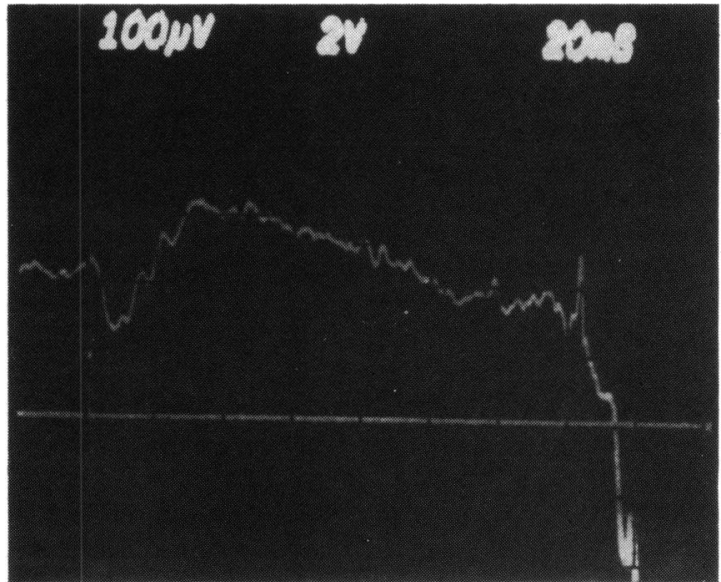

Fig. 3 Preoperative electroretinographic tracings from an eye undergoing vitrectomy alone (control).

eyes the increases in the first postoperative week were as much as $100 \%$ of baseline values.

Nine months postoperatively one eye undergoing vitrectomy alone showed a fall from a baseline value of 180 to 130 microvolts ( $\Delta B=-50$ microvolts); three eyes undergoing vitrectomy and silicone oil injection at the same time showed an average fall from baseline values of 13 microvolts $(\Delta B=-13)$.

Three animals were retested on a different flash unit and amplifier at 14 months, 17 months, and 20 months postoperatively. Comparisons are valid only between eyes of the same animal and not compared with control values because of the change in equipment. In the animal tested 14 months postoperatively the $b$ wave values at I16 were: vitrectomy alone, 140 $\mu \mathrm{v}$, vitrectomy and silicone oil injection, $185 \mu \mathrm{v}$. In the animal studied 17 months postoperatively one eye had no operation $(B=80 \mu v)$; the fellow eye had undergone vitrectomy and silicone oil injection $(B=80 \mu v)$. In the animal studied 20 months post-

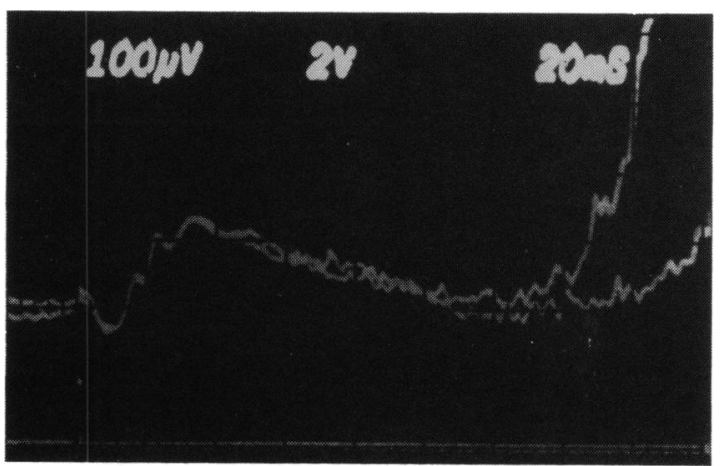

Fig. 4 Four-day postoperative electroretinographic tracings from control eye in Fig. 3. 


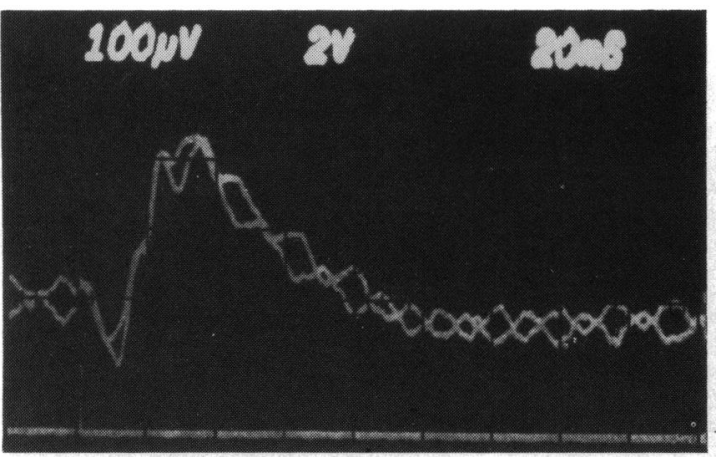

Fig. 5 Four-week postoperative electroretinographic tracings from control eye in Fig. 3.

operatively, b wave values were: vitrectomy alone, $90 \mu \mathrm{v}$, vitrectomy and silicone oil injection, $100 \mu \mathrm{v}$.

Studies of histology by light microscopy showed no alteration of normal retinal pigment epithelium architecture in either control or silicone injected eyes four days after surgery. In the specimen studied two months postoperatively an eye without silicone injection was found to be largely without abnormalities, except for one section, which showed a focal area of photoreceptor loss and outer nuclear layer loss. The fellow eye had cataract and retinal detachment.

In specimens studied three months postoperatively the eye undergoing vitrectomy showed no alterations, while that having undergone silicone injection showed only a few lipid droplets at the level of the retinal pigment epithelium without any disruption of retinal architecture (Fig. 9).

Six months postoperatively one animal showed no abnormalities in either eye. In a second animal the control eye had occasional pyknotic nuclei in the photoreceptor layer resembling late stages of photic damage. Other eyes had some loss of outer and inner segments, intraretinal macrophages, and mild metaplasia of the retinal pigment epithelial cells. Most

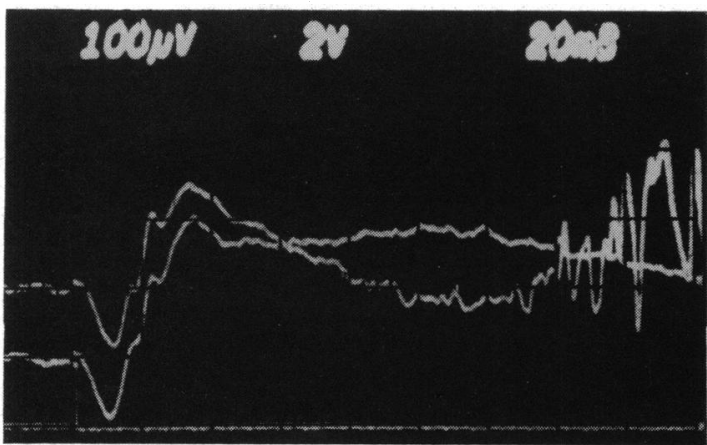

Fig. 6 Preoperative electroretinographic tracings from fellow eye undergoing vitrectomy and silicone oil injections.

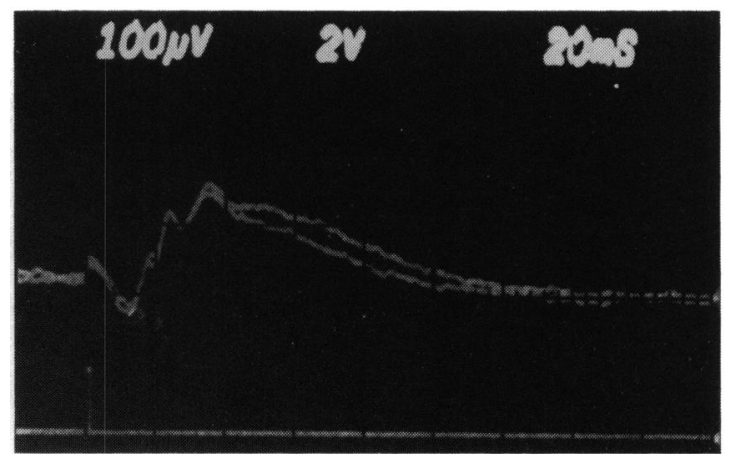

Fig. 7 Four-day postoperative electroretinographic tracings from fellow eye in Fig. 6.

areas remained normal. In the eye with silicone injection the superior retina and visual streak appeared normal. There was one hypopigmented area, however, which showed loss of outer segments and invasion of the retina by pigmented macrophages. The retinal pigment epithelium tended to be bunched together in this area, together with an increase in the pigment content of the cells (Fig. 10).

In the animal killed nine months postoperatively the control eye had developed a retinal detachment, and there was a prominent loss of photoreceptors. The eye containing silicone was normal, except for a slight random increase in pigmentation in the retinal pigment epithelium in some areas of the superior retina. Despite inability to remove cortical vitreous in the rabbit, essentially no vitreous was histologically detectable in superior areas of the retina where silicone oil had been in constant contact with the retinal surface in any of the specimens.

\section{Discussion}

Surgical intervention into the vitreous cavity produces a fall in the amplitude of both $a$ and $b$ waves

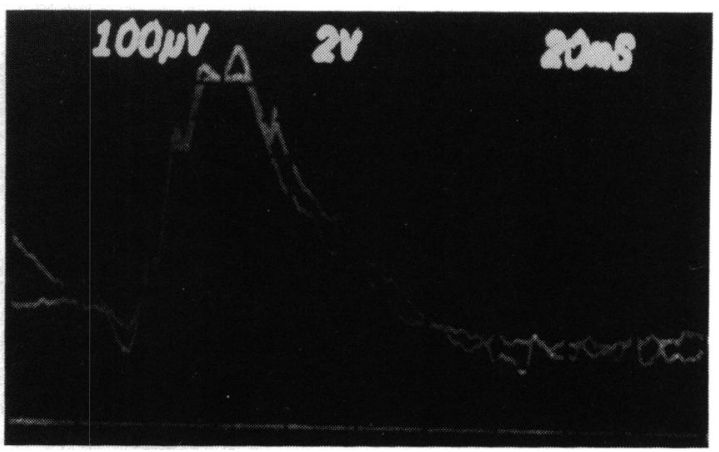

Fig. 8 Four-week postoperative electroretinographic tracings from fellow eye in Fig. 6. 
Fig. 9 Retina maintains normal architecture three months after vitrectomy and silicone oil injection without evidence of residual vitreous. (Haematoxylin and eosin, $\times 125)$.

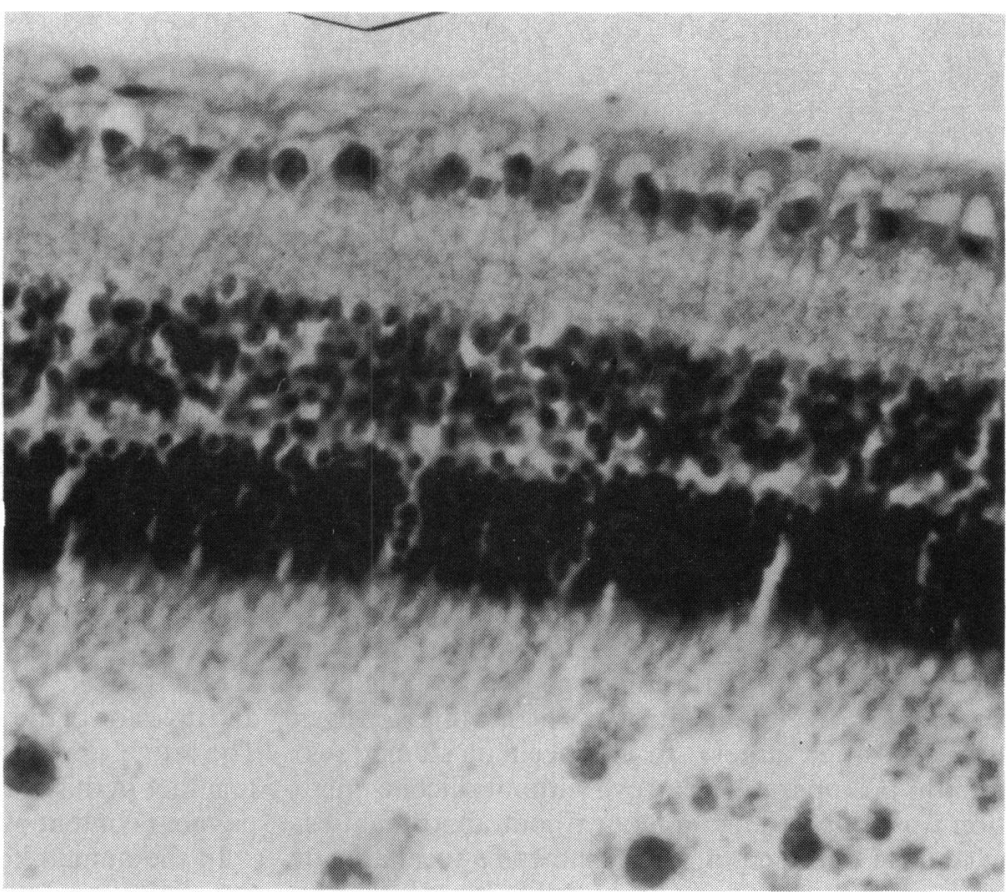

of the electroretinogram, ${ }^{23}{ }^{24} \mathrm{a}$ finding confirmed by the control eyes in our study. On day 2 eyes with vitrectomy alone showed a decrease of $31 \%$ of b wave amplitude, while those with vitrectomy and silicone

oil injection showed an average decline of $39 \%$, comparable with results after surgical injury alone reported by Declerq and colleagues..$^{23}$ The degree of initial decline has been found in part to vary with

Fig. 10 Retina maintains normal architecture six months after vitrectomy and silicone oil injection without evidence of residual vitreous on retinal surface. (Luxol fast blue, $\times 125$ ).

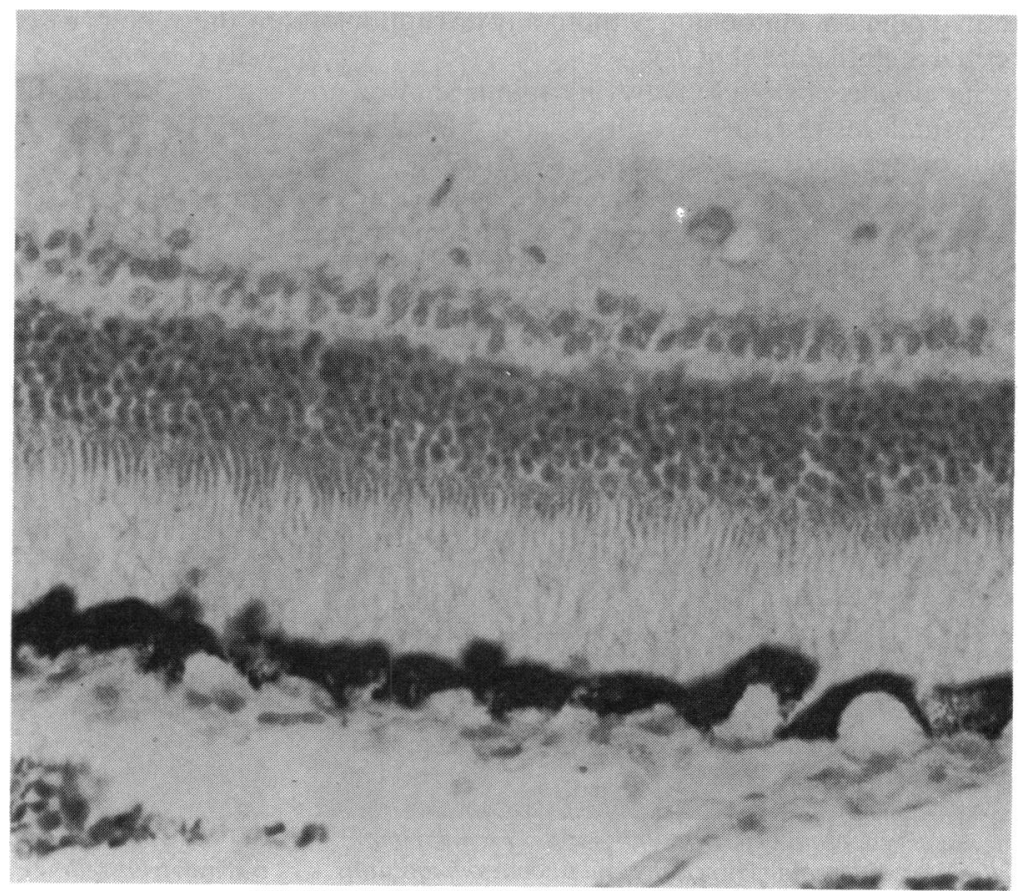


surgical irrigating solution, ${ }^{24}$ though decreases in b wave amplitude may occur with scleral puncture alone without intraocular irrigations. ${ }^{23}$

Eyes with and without silicone showed equal and parallel initial declines in $b$ wave amplitudes, followed by increases to levels greater than baseline values. The initial decline can now be attributed to surgical intervention alone rather than to an effect of silicone in the vitreous cavity, as previously concluded. ${ }^{9}$

With surgical intervention alone, ${ }^{2324}$ or with silicone implantation, ERG $a$ and $b$ wave values usually return to normal between two and seven days after surgery. Armaly ${ }^{5}$ has also previously demonstrated normal ERGs after prolonged implantations of silicone in the vitreous cavity. Prolonged reduction or increasing loss of amplitude may be produced by implanting a metallic foreign body in the vitreous cavity or by injecting blood..$^{25}$

The increase in $b$ wave amplitude to values greater than normal has not been previously reported and is not readily explained. In experimental models a and b wave amplitudes may be increased by lowering temperature and increasing $\mathrm{pH} .{ }^{26}$ Alterations in ionic content of the solution bathing the retina may also produce alterations in wave forms. ${ }^{26}{ }^{27}$ Such local changes may occur in vitrectomised eyes but have not been systematically correlated with electroretinographic changes. Increases in vitreous calcium, magnesium, and protein concentration occur after vitrectomy, while vitreous potassium is decreased..$^{28}$ These changes usually revert to normal, however, more quickly than does the increased $b$ wave amplitude we noted.

Lee and associates ${ }^{9}$ demonstrated early histological changes in rabbits and monkeys consisting of swelling in the nerve fibre layer and hydropic vacuolisation of ganglion cells in the retina. There were early cloudy swelling changes in the rods and cones. These findings reverted to normal in the late stages, leaving only a reduction in the ganglion cells and a commensurate reduction of the thickness of the ganglion cell layer. Although these latter changes were noted to be associated with a suppressed ERG, the ganglion cell layer is not thought to make any contribution to the ERG; therefore there cannot be a causal relationship between these two findings.

Mukai and colleagues ${ }^{10}$ noted spaces within the retina shortly after silicone injection, which he interpreted as silicone particles. Phospholipids were noted at the vitreoretinal interface. Later studies identified empty spaces in the inner segment of the retina. ${ }^{11}$

Our findings of little change in the histology on light microscopy confirm earlier work by Armaly and LaBelle and Okun. ${ }^{6}$ Neither of these previous investigations demonstrated long-term damage to the retina by intravitreal silicone oil. Early retinal oedema was noted in both control and silicone injected eyes by LaBelle and $\mathrm{Okun}^{6}$ and was attributed to surgical trauma.

Pathological studies in man after silicone oil injection are limited. ${ }^{29-33}$ One detailed study by Manschot ${ }^{29}$ demonstrated degeneration in a retina that was chronically detached, while two additional studies ${ }^{3031}$ noted changes in eyes which had been phthisical for five years. Studies on an eye which had been phthisical for only a few months ${ }^{8}$ showed only a few vacuoles within the nerve fibre layer, with a homogeneous layer of moderate electron density overlying the inner limiting membrane. The intraretinal globules were contained within macrophages and glial cells rather than affecting the neurosensory elements of the retina. Okun ${ }^{17}$ noted that studies of 13 eyes studied pathologically after silicone oil injection showed only minimal reaction to the silicone itself, but these cases were not recorded in detail.

No evidence of clinical silicone retinopathy has ever been demonstrated..$^{78161731}$ On the contrary, long-term follow-up studies have demonstrated maintenance of vision for three $,{ }^{18} 10,{ }^{14}$ and $12{ }^{17}$ years once the retina is reattached without evidence of loss of vision attributable to silicone toxicity. The absence of significant decline in the electroretinographic response from animal eyes undergoing vitrectomy and silicone oil injection supports the concept that the retina tolerates intraocular silicone well and does not provide evidence for a functional silicone retinopathy.

This study was supported by a grant from the Wisconsin Council for the Prevention of Blindness.

\section{References}

1 Cibis PA, Becker B, Okun E, Canaan S. The use of liquid silicone in retinal detachment surgery. Arch Ophthalmol 1963; 68: 590-9.

2 Cibis PA. Vitreous transfer and silicone injections. Trans Am Acad Ophthalmol Otolaryngol 1964; 68: 983-7.

3 Stone W Jr. Alloplasty in surgery of the eye. N Engl J Med 1958; 258: 486-502.

4 Levine AM, Ellis RA. Intraocular liquid silicone implants. Am J Ophthalmol 1963; 55: 939-43.

5 Armaly MF. Ocular tolerance to silicones. I. Replacement of aqueous and vitreous by silicone fluids. Arch Ophthalmol 1962; 68: $390-5$.

6 LaBelle P, Okun E. Ocular tolerance to liquid silicone: an experimental study. Am J Ophthalmol 1972; 7: 199-204.

7 Watzke RC. Silicone retinopoiesis for retinal detachment. Arch Ophthalmol 1967; 77: 185-96.

8 Leaver PK, Grey RHB, Garner A. Silicone oil injection in the treatment of massive periretinal proliferation. II. Complications in 93 eyes. Br J Ophthalmol 1979; 63: 361-7.

9 Lee P, Donovan RH, Mukai N, Schepens CL, Freeman HM. Intravitreous injection of silicone: an experimental study. I. Clinical picture and histology of the eye. Ann Ophthalmol 1969; 1: $15-25$. 
10 Mukai N, Lee P, Schepens CL. Intravitreous injection of silicone: an experimental study. II. Histochemistry and electron microscopy. Ann Ophthalmol 1972; 4: 273-87.

11 Mukai N, Lee P, Oguri M, Schepens CL. A long term evaluation of silicone retinopathy in monkeys. Can J Ophthalmol 1975; 10: 391-402.

12 Scott JD. The treatment of massive vitreous retraction by separation of preretinal membranes using liquid silicone. Mod Probl Ophthalmol 1975; 15: 285-90.

13 Scott JD. Equatorial giant tears affected by massive vitreous retraction. Trans Ophthalmol Soc UK 1976; 96: 309-12.

14 Scott JD. A rationale for the use of liquid silicone. Trans $O p h$ thalmol Soc UK 1977; 97 : 235-7.

15 Scott JD. A rationale for the use of liquid silicone in retinal detachment surgery. In: Shimuzu K, ed. International Congress Series 450 XXII Concilium Ophthalmologicum, Kyoto. Amsterdam-Oxford: Excerpta Medica, 1978.

16 Okun E. Intravitreal surgery utilizing liquid silicone. A long term follow up. Trans Pac Coast Otoophthalmol Soc 1968; 49: 141-59.

17 Okun E. The current status of silicone oil (analysis of long term successes). In: Irvine AR, O'Malley C, eds. Advances in vitreous surgery. Springfield: Thomas, 1976: 518-22.

18 Grey RHB, Leaver PK. Silicone oil in the treatment of massive periretinal proliferation. I. Results in 105 eyes. Br J Ophthalmol 1979; 63: 355-60.

19 Zivonjnovic R, Mertens DAE, Baavsma GS. Das flüssige Silikon in der Amotiochirurgie. Klin Monatsbl Augenheilkd 1981; 179: 17-22.

20 Meredith TA, Lindsey DT, Goldman AI. Electroretinographic evaluation after vitrectomy and silicone oil injection. Invest $O p h$ thalmol Visual Sci 1981; 20 (suppl): 199.
21 Ober RA, Blanks JC, Ogden TE, et al. Retinal tolerance to liquid silicone. Retina $1983 ; 3: 77-85$.

22 Abrams GW, Topping T, Machemer R. An improved method for practice vitrectomy. Arch Ophthalmol 1978; 96: 521-5.

23 Declerq SS, Meredith CA, Rosenthal AR. Experimental siderosis in the rabbit. Arch Ophthalmol 1977; 95: 1051-8.

24 Moorhead LC, Redburn DA, Merritt J, Garcia CA. The effects of intravitreal irrigation during vitrectomy on the electroretinogram. Am J Ophthalmol 1979; 88: 239-45.

25 Mandelbaum S, Cleary PE, Ryan SJ, Ogden TE. Bright-flash electroretinography and vitreous hemorrhage. Arch Ophthalmol 1980; 98: 1823-8.

26 Winkler BS. The electroretinogram of the isolated rat retina. Vision Res 1972; 12: 1183-97.

27 Winkler BS, Simson V, Banner S. Importance of bicarbonate in retinal function. Invest Ophthalmol Visual Sci 1977; 16: 766-8.

28 Killey FP, Edelhauser HG, Aaberg TA. Intraocular fluid dynamics: measurements following vitrectomy and intraocular sulfur hexafluoride. Arch Ophthalmol 1980; 98: 1448-52.

29 Manschot WA. Intravitreal silicone injection. Adv Ophthalmol 1978; 36: 197-207.

30 Sugar HS, Okamura ID. Ocular findings in six years after intravitreal silicone injection. Arch Ophthalmol 1976; 94: 612-5.

31 Blodi FC. Injection and impregnation of liquid silicone in ocular tissues. Am J Ophthalmol 1971; 71: 1044-51.

32 Watzke RC. Silicone retinopoeisis for retinal detachment: a pathologic report. Surv Ophthalmol 1967; 12: 333-7.

33 Cockerham WD, Schepens CL, Freeman HM. Silicone injection in retinal detachment. Arch Ophthalmol 1970; 83: 704-12. 